\title{
Time Perception in Mild Cognitive Impairment: Interval Length and Subjective Passage of Time
}

\author{
Sara Coelho, ${ }^{1}$ Manuela Guerreiro, ${ }^{1}$ Catarina Chester, ${ }^{1}$ Dina Silva, ${ }^{2}$ João Maroco, ${ }^{3}$ Miguel Coelho, ${ }^{1}$ Fabio Paglieri, ${ }^{4}$ \\ AND Alexandre de Mendonça ${ }^{1}$ \\ ${ }^{1}$ Institute of Molecular Medicine and Faculty of Medicine, University of Lisbon, Lisbon, Portugal \\ ${ }^{2}$ Cognitive Neuroscience Research Group, Department of Psychology and Educational Sciences and Centre for Biomedical Research (CBMR), University \\ of Algarve, Faro, Portugal \\ ${ }^{3}$ William James Center for Research, ISPA-IU, Lisboa, Portugal \\ ${ }^{4}$ Institute for Cognitive Sciences and Technologies of the CNR, Rome, Italy
}

(Received December 19, 2015; Final Revision May 15, 2016; Accepted June 9, 2016)

\begin{abstract}
Objectives: Patients with mild cognitive impairment (MCI) may have difficulties in time perception, which in turn might contribute to some of their symptoms, especially memory deficits. The aim of this study was to evaluate perception of interval length and subjective passage of time in MCI patients as compared to healthy controls. Methods: Fifty-five MCI patients and 57 healthy controls underwent an experimental protocol for time perception on interval length, a questionnaire for the subjective passage of time and a neuropsychological evaluation. Results: MCI patients presented no changes in the perception of interval length. However, for MCI patients, time seemed to pass more slowly than it did for controls. This experience was significantly correlated with memory deficits but not with performance in executive tests, nor with complaints of depression or anxiety. Conclusions: Memory deficits do not affect the perception of interval length, but are associated with alterations in the subjective passage of time. (JINS, 2016, 22, 755-764)
\end{abstract}

Keywords: Cognitive decline, Time experiences, Time interval, Perceived speed of time judgments, Memory, Aging

\section{INTRODUCTION}

The number of patients with cognitive complaints has been rising as a consequence of the increasing aging of the population. Clinicians have especially focused on patients diagnosed with mild cognitive impairment (MCI), because they carry a high risk for developing dementia in the ensuing few years. According to the original Mayo criteria, the presence of memory impairment in patients with preserved general cognitive function and independence in basic activities of daily living represent an increased risk of progression to Alzheimer's disease (AD; Petersen et al., 1999). Patients in the initial stages of AD often refer to losing track of dates, seasons, and the passage of time (Alzheimer's Association, 2009). Difficulties in placing events in the correct temporal framework may compromise orientation and daily planning, since time perception is a crucial component of everyday decisions and goal-oriented behaviors

Correspondence and reprint requests to: Sara Coelho, Laboratory of Neuroscience, Institute of Molecular Medicine, Av Prof Egas Moniz, 1649-028 Lisboa, Portugal. E-mail: arascoelho@gmail.com
(Buhusi \& Meck, 2005; Mangels \& Ivry, 2001). However, there has been scarce research on time perception in patients with MCI.

Time perception comprises at least two main subjective time experiences: interval length judgments and the subjective passage of time judgments (Block, 1990; Sucala, Scheckener \& David, 2010; Wearden, 2005). Perception of interval length concerns the subjective evaluation of a certain duration and has been mainly approached using two different paradigms: prospective tasks (participants are told in advance that they will have to estimate a time interval) and retrospective protocols (participants are not told in advance that they will have to estimate a time interval), as well as two distinct methods of investigation, verbal time estimation (participants have to verbally estimate the duration of different intervals) and verbal time production (participants have to produce different interval durations). Experiencing the subjective passage of time involves another type of time experience that relates to the perceived speed of the time course: this is typically assessed via first-person reports on the subject's own feelings on time passage (Friedman \& Janssen, 2010; Wearden, 2005). 
Recent studies on time perception have often been based on the internal clock model. This model supposes that each individual has an internal clock which is moving slower or faster according to how temporal judgments about interval length approach or deviate from real measured time (Droit-Volet \& Wearden, 2003; Grodin, 2010). An oscillatory pacemaker constantly emitting pulses at the same rate composes the clock, which processes information along three stages.

First, at the clock stage, the pulses enter into an accumulator when the switch is open. Attention plays here an important role; for instance, inattention slows down the internal clock, producing a lower number of pulses. The memory stage follows. After the switch is closed, the pulses gated in the accumulator, which represent the current time, are stored in the working memory system for comparison with the values contained in reference memory, that is, the long-term memory for pulses accumulated in the past. Finally, in the decisional stage, the values present in working memory are compared with those stored in the reference memory, allowing a decision to be made on the perception of time.

The main advantage of this model is to identify the sources of individual and pathophysiological differences in time perception and relate them to neuropsychological data (Allman \& Meck, 2012; Nichelli, 1993). It is important to note that, according to this internal clock model, working memory and executive functions are determinant mechanisms for time perception, due to the role of attentional shifts in determining the perceived speed of the internal clock (Heinik \& Ayalon, 2010; Papagno, Allegra, \& Cardaci, 2004; Pouthas \& Perbal, 2004). Remarkably, episodic memory and prospective and retrospective memory are also thought to be necessary for time perception (Graf \& Grodin, 2006; Kinsbourne \& Hicks, 1990; Mimura, Kinsbourne, \& O'Conner, 2000; SchmitterEdgecombe \& Rueda, 2008). We would then expect that a population with memory deficits, such as MCI patients, would present significant impairments in time perception. However, previous studies failed to find a clear correlation between MCI and time perception deficits.

We posit that this lack of results is due to exclusive emphasis on interval length perception, a cognitive skill that does not necessarily matters for the kind of long-term temporal projection involved in episodic and prospective memory. In studies on perception of interval length, there was no significant difference between MCI patients and controls in prospectively evaluating short time intervals, that is, in between 10 and $60 \mathrm{~s}$ (Rueda \& Schmitter-Edgecombe, 2009). Another study evaluated self-estimation of performance time (Heinik \& Ayalon, 2010): participants were asked to estimate retrospectively the actual duration of the interview (the real duration was 25 to $30 \mathrm{~min}$ ). Selfestimation of performance time versus actual performance time was not impaired in MCI patients when compared to participants without cognitive impairment.

The results of these two experimental studies suggest that MCI patients have no alterations in the perception of interval length. Alternatively, methodological limitations of the chosen tasks might have hindered the detection of those alterations.
First of all, it would be important to assess both the prospective paradigm and the retrospective paradigm, using the verbal time estimation as well as the verbal time production methods to estimate or produce the duration of empty time intervals to evaluate more extensively the perception of the interval length. Empty time intervals were preferred in this methodology over filled intervals since non-temporal tasks would interfere with working memory and attention functions and, therefore, reduce the accuracy in temporal perception (Coelho et al., 2004; Fortin, Rosseau, Bourque, \& Kirouac, 1993).

In contrast, the subjective experience of time passage in MCI patients has not been studied in the literature, to the best of our knowledge. This, we argue, constitutes a major limitation of current studies, since it is precisely the subjective experience of the passing of time that is likely to have a significant impact on memory deficits. If the perception of the passage of time is dependent upon memory (Bergson, 1889), we may speculate that patients with MCI might have a hindered perception of time passage. It is also possible that patients with MCI are less able to engage in novel activities requiring emotional or intellectual commitment, and thereby believe the time is passing more slowly (Sucala et al., 2010).

To test this hypothesis, as well as to confirm the lack of differences in interval length judgements, in the present study we analyzed time perception in MCI patients with respect to interval length and passage of time judgments on short durations (seconds, minutes), using both the prospective paradigm and the retrospective paradigm, both for verbal time estimation and for verbal time production (Coelho et al., 2004). Time perception of longer intervals (hours, days, weeks) and the perceived speed of time passage in MCI were also assessed. An interesting aspect was to see whether time perception in patients with MCI would be better or worse than in controls, that is, closer to or farther from the objectively measured temporal values.

\section{METHODS}

\section{Participants}

MCI patients were recruited at a dementia outpatient clinic and a memory clinic, both in Lisbon. Controls were volunteers with no cognitive complaints from senior universities in Lisbon. The study was approved by the ethical committee of the Faculty of Medicine/ Santa Maria Hospital in Lisbon. The participants were informed of the experimental protocol and gave their written consent.

\section{Inclusion Criteria for the MCI Group}

The inclusion criteria for the diagnosis of $\mathrm{MCI}$ were adapted from Petersen et al., 1999, with an emphasis on amnestic MCI: (1) presence of memory complaints; (2) abnormal memory function, documented by the Logical Memory A below education and age adjusted values for the Portuguese population $(1 S D)$ or who lost $>3$ points after delay. Logical Memory is a 
subtest of the Bateria de Lisboa para Avaliação das Demências (BLAD) (Garcia, 1984; Guerreiro, 1998), a neuropsychological battery designed to evaluate multiple cognitive domains and validated for the Portuguese population; (3) normal general cognitive function, determined by the Mini Mental State Examination (MMSE, Folstein, Folstein, \& McHugh, 1975) within normal values for the Portuguese population. The Portuguese version of the test, adapted from Guerreiro et al. (1994), was used; (4) no impairment or minimal impairment in activities of daily living determined by the Instrumental Activities of Daily Living Scale (IADL, Lawton \& Brody, 1969), that is, no more than one item from the IADL scale was abnormal. The Portuguese version, developed in the context of the LADIS project, was used (Pantoni et al., 2005).

\section{Inclusion Criteria for the Control Group}

The control participants had: (1) no memory complaints; (2) normal memory function, documented by the Logical Memory subtest of the Bateria de Lisboa para Avaliação das Demências (BLAD); (3) a Mini-Mental State Examination (MMSE) with normal values for the Portuguese population; (4) normal scores on the IADL scale, that is to say, no item from the IADL scale was abnormal.

\section{Inclusion Criteria for Both Groups}

Native Portuguese speakers; education $\geq 4$ years; age $>45$ years old.

\section{Exclusion Criteria for Both Groups}

(1) Dementia, according to DMS-IV-TR (American Psychiatric Association, 1994). (2) The presence of major depression according to DSM-IV-TR or serious depressive symptoms, indicated by a score $>10$ points on the 15 -item Geriatric Depression Scale ${\left(G_{D S}\right.}_{15}$, Yesavage et al., 19821983). The Portuguese version of the test was used, adapted from Barreto, Leuschner, Santos, and Sobral (2008). (3) Neurological disorders (Parkinson's disease, stroke, brain tumor, significant head trauma or epilepsy), psychiatric conditions (such as autism or schizophrenia), or uncontrolled medical illness (hypertension, metabolic, endocrine, toxic or infectious diseases) able to interfere with cognitive performance. (4) Psychoactive medications with possible influence on cognitive performance. (5) History of alcohol or drug abuse. (6) Sensory deficits likely to interfere with assessment. (7) Participants with MMSE below education-adjusted values for the Portuguese population were excluded $(<23$ for equal or less than 11 years of education, $<28$ for more than 11 years of education).

\section{Assessments}

All the participants were submitted to the Portuguese version of the following instruments:

\section{Neuropsychological Measures}

(1) Tests that evaluate immediate memory (Digit Span forward), working memory (Digit Span backward), and verbal memory (Logical Memory). These tests are from the Bateria de Lisboa para Avaliação das Demências (BLAD) (Garcia, 1984; Guerreiro, 1998). (2) Stroop test (STROOP, MacLeod, 1991). The Stroop test assesses executive functions, namely selective attention. The Portuguese version of the test, adapted from Castro et al. (2000), was used. (3) Trail Making Test (TMT, Reitan, 1958). The TMT evaluates executive functions, namely attention switching, planning and internal ordering. The Portuguese version of the test, adapted from Fernandez and Marcopulos (2008), was used. (4) Subjective Memory Complaints (SMC, Schmand, Jonker, Hooijer, \& Lindeboom, 1996). The SMC is a questionnaire that assesses memory complaints. The Portuguese version of the test, adapted from Ginó et al. (2008), was used. (5) Prospective and Retrospective Memory Questionnaire (PRMQ, Smith, Della Sala, Logie, \& Maylor, 2000). This is a tool to measure self-reports of prospective and retrospective memory. A Portuguese version was used (based on Sara da Câmara's master thesis, unpublished).

\section{Emotional Status Measures}

(1) State-Trait Anxiety Inventory (STAI, Spielberger, Gorssuch, Lushene, Vagg, \& Jacobs, 1983). The STAI is a questionnaire that evaluates the trait and the state of anxiety. The Portuguese version of the test, adapted from Silva (2006), was used.

(2) The Geriatric Depression Scale $\left(\mathrm{GDS}_{15}\right)$. The GDS is a questionnaire that evaluates presence and severity of depression.

\section{Experimental Measures of Time Perception}

To evaluate time perception, all participants were asked to complete the following tasks: an experimental protocol on interval length and a questionnaire for the subjective passage of time.

The experimental protocol for time perception on interval length (Coelho et al., 2004) is a test in which participants first have to prospectively estimate empty intervals signaled by auditory beeps (of $7 \mathrm{~s}, 32 \mathrm{~s}$, and $58 \mathrm{~s}$ duration); then participants have to prospectively produce empty intervals signaled by auditory beeps (again, of $7 \mathrm{~s}, 32 \mathrm{~s}$, and $58 \mathrm{~s}$ duration). For each task, every duration was repeated three times in a pseudorandom order, so that for both estimation and production tasks nine trials were performed. Participants were told to start the internal counting of seconds after they heard the first beep and to stop counting when they heard the second beep (in case of the estimation task) or to tell the examiner when they had reached the target duration (in case of the production task). They were specifically instructed not to count aloud nor to perform any digital counting, or use any body rhythm to help in the estimation. In addition, 
participants were asked, retrospectively, to estimate the time elapsed while they were drawing a clock and during the neuropsychological evaluation. Participants are said to have a faster internal clock if they overestimate and underproduce time intervals, that is, their estimations are above actual duration while their productions are below it. On the contrary, participants are said to have a slower internal clock if they underestimate and overproduce time intervals.

The questionnaire for the subjective passage of time (Friedman \& Janssen, 2010) is a tool that assesses the subjective impressions of longer time intervals, that is, whether participants experience time as passing slowly or quickly. The test consists of two parts. The first part has six questions concerning how participants experience the speed at which time seems to pass (these questions are included in the caption of Figure 1): each item is to be rated on a five-point scale, ranging from very slow $(-2)$ to very fast $(+2)$. Two questions ask about our time experience in the present, the first concerning our typical experience of time ("How long does time usually pass for you?") and the second referring to a specific time perception ("How long do you expect the next hour to pass?"). The other four questions cover the perception of the past, focusing on various time intervals (last week, last month, last year, and the past 10 years).

The global perceived speed of time is measured as a composite speed-of-time score, which is the sum of the six

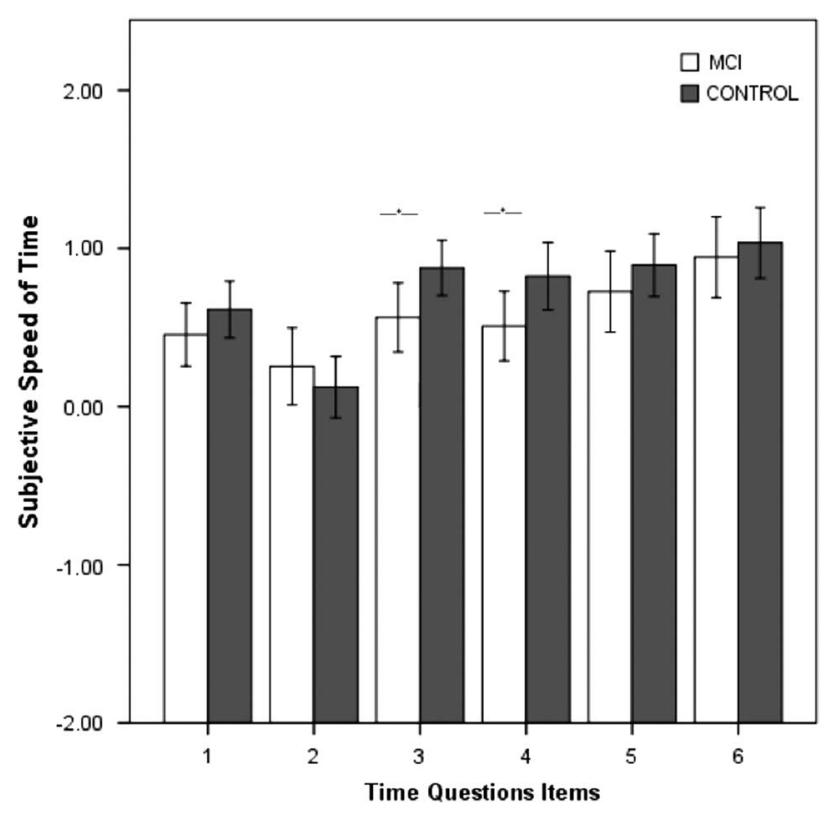

Fig. 1. Perceived speed of time. The ratings of the perceived speed of time questionnaire are shown (mean $\pm S E$ ). Items: 1. How fast does time usually pass for you? 2. How fast do you expect the next hour to pass? 3. How fast did the previous week pass for you? 4. How fast did the previous month pass for you? 5. How fast did the previous year pass for you? 6 . How fast did the previous 10 years pass for you?). Participants rated the statements on a five-point scale that ranged from 'very slowly? [-2] to 'very fast' [2]. *Statistically significant; Independent samples Student's $t$ tests. questionnaire items (Friedman \& Janssen, 2010). The second part includes 11 statements about the subject's experience of time (see the leftmost column of Table 3), which the participants have to rate on a seven-point scale ranging from "strongly disagree" (-3) to "strongly agree" $(+3)$. The statements of the subjective experience of time were conceived to evaluate the impact of subjective impressions of life experience on the subjective feelings of time passage, focusing on: (a) the effects of recent life changes (items 1-4), since it is thought that more activity and more life experiences would give the impression that time is moving at a fast pace; (b) forward telescoping (items 5-7), that is, the temporal displacement of a distant event could make it look more recent than it actually was, thus conveying the impression that the time is passing quickly; and (c) the amount of pressure and rushing one experiences in life (items 8-11), since feelings like being always busy or never having enough time to get things done usually sustain the sensation that time is fleeting.

\section{Statistical Analysis}

Sample size was estimated from a power analysis using the Power and Precision (v.4; BioStat; Englewood, NJ) software. For previous sample statistical estimates required for sample size calculation, preliminary data from 20 participants (10 MCI and 10 controls) was used. The values obtained for estimation of time $(7 \mathrm{~s})$ were $17.1 \pm 6.3 \mathrm{~s}$ in MCI patients and $13.7 \pm 3.5 \mathrm{~s}$ in controls. With such estimates, to detect a significant difference between MCI and controls, assuming a power $=90 \%, \alpha=0.05$, and two-tailed Student's $t$ test, 100 participants (50 MCI and 50 controls) would be required.

Demographic, clinical and neuropsychological data were compared between the 2 groups, MCI and controls, with the Student's $t$ test for numerical variables and $\chi^{2}$ for categorical variables.

Analysis on time perception using the prospective paradigm and short intervals was performed with a mixed effects repeated-measures analysis of variance (ANOVA). Patients with MCI and controls were used to evaluate betweensubjects effects, while time $(7 \mathrm{~s}, 32 \mathrm{~s}, 58 \mathrm{~s})$ and order of presentation $\left(1^{\text {st }}, 2^{\text {nd }}, 3^{\text {rd }}\right)$ were used to evaluate within-subjects effects. When significant effects were detected with the ANOVA, Students' $t$ tests were performed with Bonferroni corrections, to identify the groups differences. Effect sizes were estimated by the partial eta squared $\left(\eta_{\mathrm{P}}^{2}\right)$ calculated by SPSS. Differences in the retrospective paradigm on long intervals between the 2 groups were evaluated with the Student's $t$ test. The differences in the perceived speed of time between the two groups were assessed using a composite speed-of-time score (Friedman \& Janssen, 2010). Differences in individual items scores were additionally explored with the same test. The scores of the statements about the subjective experience of time were also compared using the Student's $t$ test. The correlational analysis between neuropsychological variables and the composite speed-of-time scale score was performed with the Pearson's correlation. 
Statistical analyses were performed using SPSS for Windows (SPSS 19; SPSS Inc., Chicago, IL). Effects with $p$ values $<.05$ were considered statistically significant.

\section{RESULTS}

One hundred twelve participants, 57 controls and $55 \mathrm{MCI}$ patients, were submitted to neuropsychological evaluation, an experimental protocol for time perception on both short intervals and long intervals and a questionnaire on subjective passage of time. There were no statistically significant differences in age, education, gender, and activity/retirement status between the two groups (Table 1).

\section{Neuropsychological Characteristics}

As expected, the MCI participants had lower MMSE scores, worse performances in cognitive tests, namely memory (Logical Memory, Digit Span) and executive functions tests (Trail A and B, Stroop test), and presented more subjective memory complaints (SMC, PMRQ) as well as anxiety (STAI) and depressive (GDS) symptoms than controls (Table 1).

\section{Time Perception on Interval Length}

In the prospective paradigm on short intervals, the time estimates were above the actual times, and the time productions were under the actual times, for the three times considered ( $7 \mathrm{~s}, 32 \mathrm{~s}, 58 \mathrm{~s})$, in both MCI patients and controls, as widely known from previous studies (Table 2). There were no significant differences between MCI patients and controls on time estimate (repeated measures ANOVA, $F$ $\left.(1,110)=0.138 ; p=.711 ; \eta_{p}^{2}=0.0013\right)$. Furthermore, no interactions between the diagnostic group and the time $(7 \mathrm{~s}$, $32 \mathrm{~s}, 58 \mathrm{~s}$; repeated measures ANOVA, $F(2,220)=0.072$; $\left.p=.930 ; \eta_{\mathrm{p}}^{2}=0.0006\right)$ and the diagnostic group and the order of presentation $\left(1^{\text {st }}, 2^{\text {nd }}, 3^{\text {rd }}\right.$; repeated measures ANOVA, $\left.F(2,220)=0.971 ; p=.380 ; \eta_{\mathrm{p}}^{2}=0.0087\right)$ were found on average time estimates. There were also no significant differences between MCI patients and controls on time production (repeated measures ANOVA, $F$ $\left.(1,110)=0.043 ; p=.837 ; \eta^{2}=0.0003\right)$. No interactions between the diagnostic group and the time $(7 \mathrm{~s}, 32 \mathrm{~s}, 58 \mathrm{~s}$; repeated measures ANOVA, $F(2,220)=0.021 ; p=.980$, $\left.\eta_{\mathrm{p}}^{2}=0.0001\right)$ and the diagnostic group and the order of presentation $\left(1^{\text {st }}, 2^{\text {nd }}, 3^{\text {rd }}\right.$; repeated measures ANOVA, $F$ $\left.(2,220)=1.190 ; \mathrm{p}=.306 ; \eta_{\mathrm{p}}^{2}=0.0107\right)$ were found on average time production.

Regarding the retrospective paradigm on long intervals, there were no significant differences in the estimates of the time required to draw the clock and the duration of the interview between MCI patients and controls (Table 2).

\section{Perception on Subjective Passage of Time}

Regarding the perceived speed of time passage, the composite speed-of-time scale score was significantly lower in MCI patients $(0.58 \pm 0.9)$ as compared to controls $(0.73 \pm 0.8$; $t(670)=-2.341 ; p=.02$; Student's $t$ test $)$, meaning that the MCI patients reported the time to be passing slower. The individual scores for the 6 time questions are shown in Figure 1. In all the questions, except question 2 that relates to the anticipated speed of time in the next hour, the MCI

Table 1. Demographic and neuropsychological characterization

\begin{tabular}{|c|c|c|c|}
\hline & $\operatorname{MCI}(n=55)$ & CONTROL $(n=57)$ & $p$-Value \\
\hline AGE, years, mean $(S D)$ & $70.9(8.9)$ & $67.6(8.5)$ & $.05^{\mathrm{a}}$ \\
\hline EDUCATION, years, mean $(S D)$ & $10.9(4.4)$ & $11.1(4.5)$ & $.74^{\mathrm{a}}$ \\
\hline GENDER, female/male, $n$ & $36 / 19$ & $35 / 22$ & $.66^{\mathrm{b}}$ \\
\hline ACTIVITY, active/retired, $n$ & $8 / 47$ & $7 / 50$ & $.73^{\mathrm{b}}$ \\
\hline MINI-MENTAL STATE EXAMINATION, mean $(S D)$ & $27.2(2.2)$ & $29.1(1.1)$ & $<.01^{\mathrm{a}}$ \\
\hline LOGICAL MEMORY A (immediate recall), mean $(S D)$ & $7.1(3.3)$ & $15.0(4.1)$ & $<.01^{\mathrm{a}}$ \\
\hline LOGICAL MEMORY A (delayed recall), mean $(S D)$ & $5.5(3.7)$ & $15.1(4.4)$ & $<.01^{\mathrm{a}}$ \\
\hline DIGIT SPAN FORWARD, mean $(S D)$ & $5.5(0.7)$ & $5.8(1.2)$ & $.14^{\mathrm{a}}$ \\
\hline DIGIT SPAN BACKWARD, mean $(S D)$ & $3.9(1.1)$ & $4.4(1.0)$ & $.01^{\mathrm{a}}$ \\
\hline STROOP TEST (interference), mean $(S D)$ & $22.0(7.5)$ & $30.4(9.2)$ & $<.01^{\mathrm{a}}$ \\
\hline TRAIL MAKING TEST A, seconds, mean (SD) & $79.0(37.6)$ & $51.0(21.3)$ & $<.01^{\mathrm{a}}$ \\
\hline TRAIL MAKING TEST B, seconds, mean $(S D)$ & $191.1(54.7)$ & $139.9(57.9)$ & $<.01^{\mathrm{a}}$ \\
\hline SUBJECTIVE MEMORY COMPLAINTS, mean $(S D)$ & $9.6(3.6)$ & $5.5(3.0)$ & $<.01^{\mathrm{a}}$ \\
\hline PMRQ (prospective memory), mean $(S D)$ & $22.7(5.9)$ & $17.5(3.8)$ & $<.01^{\mathrm{a}}$ \\
\hline PMRQ (retrospective memory), mean $(S D)$ & $22.4(4.8)$ & $18.1(4.3)$ & $<.01^{\mathrm{a}}$ \\
\hline STATE TRAIT ANXIETY INVENTORY (trace), mean (SD) & $39.9(9.8)$ & $32.5(9.0)$ & $<.01^{\mathrm{a}}$ \\
\hline GERIATRIC DEPRESSION SCALE, mean $(S D)$ & $4.2(2.4)$ & $2.2(1.7)$ & $<.01^{\mathrm{a}}$ \\
\hline
\end{tabular}

Note. Statistically significant values are shown in bold.

andependent samples Student's $t$ tests.

${ }^{\mathrm{b}}$ Pearson chi-square test.

MCI, mild cognitive impairment; $S D$, standard deviation; PMRQ, prospective and retrospective memory questionnaire. 
Table 2. Time Perception on Interval Length

\begin{tabular}{|c|c|c|c|c|}
\hline & & MCI Mean $\pm S D$ & CONTROL Mean $\pm S D$ & $p$-Value \\
\hline \multicolumn{5}{|c|}{ PROSPECTIVE PARADIGM (SHORT INTERVALS) } \\
\hline \multirow[t]{9}{*}{ Time Estimation } & $7 \mathrm{~s}$ & $14.4 \pm 5.0$ & $14.5 \pm 5.2$ & $.71^{\mathrm{a}}$ \\
\hline & & $14.6 \pm 6.1$ & $15.3 \pm 7.1$ & \\
\hline & & $14.5 \pm 5.9$ & $15.2 \pm 6.7$ & \\
\hline & $32 \mathrm{~s}$ & $51.2 \pm 17.5$ & $54.2 \pm 17.8$ & \\
\hline & & $54.5 \pm 19.8$ & $56.2 \pm 21.0$ & \\
\hline & & $54.7 \pm 18.3$ & $55.1 \pm 19.7$ & \\
\hline & $58 \mathrm{~s}$ & $90.4 \pm 29.3$ & $93.7 \pm 31.4$ & \\
\hline & & $91.5 \pm 31.3$ & $92.4 \pm 33.5$ & \\
\hline & & $93.2 \pm 31.6$ & $93.8 \pm 34.2$ & \\
\hline \multirow[t]{9}{*}{ Time Production } & $7 \mathrm{~s}$ & $4.8 \pm 2.2$ & $4.5 \pm 2.3$ & $.84^{\mathrm{a}}$ \\
\hline & & $5.0 \pm 2.9$ & $4.9 \pm 2.3$ & \\
\hline & & $5.1 \pm 2.9$ & $5.0 \pm 2.8$ & \\
\hline & $32 \mathrm{~s}$ & $19.9 \pm 9.5$ & $18.7 \pm 9.4$ & \\
\hline & & $20.3 \pm 9.6$ & $20.2 \pm 10.1$ & \\
\hline & & $19.9 \pm 10.8$ & $20.0 \pm 10.6$ & \\
\hline & $58 \mathrm{~s}$ & $37.3 \pm 16.1$ & $36.4 \pm 16.7$ & \\
\hline & & $37.9 \pm 17.8$ & $37.3 \pm 18.2$ & \\
\hline & & $37.2 \pm 19.1$ & $36.9 \pm 18.1$ & \\
\hline \multicolumn{5}{|c|}{ RETROSPECTIVE PARADIGM (LONG INTERVALS) } \\
\hline Time to draw a clock & $\mathrm{s}$ & $-23.9^{\mathrm{b}} \pm 84.1$ & $-4.6^{\mathrm{b}} \pm 58.4$ & $.16^{\mathrm{c}}$ \\
\hline Time of the interview & $\mathrm{s}$ & $12.8^{\mathrm{b}} \pm 591.9$ & $-74.7^{\mathrm{b}} \pm 549.6$ & $.42^{\mathrm{c}}$ \\
\hline
\end{tabular}

Note. For each time, the three values concern the first, second, and third presentation of the same interval length.

${ }^{a}$ An analysis of repeated measures showed no significant statistically differences $(p<.05)$ between the Control group and the MCI group in prospective time.

${ }^{\mathrm{b}}$ The values concern the difference between real time and time estimation. Large standard deviations stem from the variability of time estimates as well as high variability of the real time, that is, participants took rather different intervals to draw a clock or had variable interview times.

'Independent samples Student's t tests.

MCI, mild cognitive impairment; $S D$, standard deviation.

patients reported the time to be passing slower, and QTI3 (How fast did the last week pass for you?, $t(110)=-2.252$; $p=.03$; Student's $t$ test), and QTI4 (How fast did the last month pass for you?, $t(110)=-2.063 ; p=.04$; Student's $t$ test) differed significantly between the two groups.

An exploratory analysis was performed on the statements about the subjective experience of time. No differences were found between MCI patients and controls except on item 2 (In the past several years my life has been quite a routine), where MCI patients described their life as being more like a routine (Table 3 ).

\section{Perceived Speed of Time and Cognition}

Since significant differences in the perceived speed of time were found between the MCI patients and control participants, it would be important to know whether the perceived speed of time could be related to performance in neuropsychological domains, as well as anxiety and depression symptoms. In control participants, no correlations were found between the speed-of-time scale score and performances in cognitive tests, subjective memory, depressive or anxiety complaints (Table 4). In contrast, in MCI patients there was a positive correlation between the speed-of-time scale score and performance on Logical Memory A (both immediate and with delay), that is, patients with more severe memory deficits felt that time was passing more slowly (Table 4). There was also a positive correlation between the speed-of-time scale score and the SMC score, that is, patients with less cognitive complaints perceived time as being slower (Table 4).

It is important to add that no statistically significant correlation was detected in MCI patients between SMC score and Logical Memory A (both immediate and with delay), meaning that patients with more cognitive deficits were not those who necessarily had more cognitive complaints. No statistically significant correlations were found in MCI patients between the speed-of-time scale score and performances in executive tests, nor with depressive or anxiety complaints (Table 4).

Of interest, item 2 of the statements about the subjective experience of time was also negatively and significantly correlated with Logical Memory A, both immediate ( $\mathrm{r}=-0.31$; $p=.02)$ and with delay $(r=-0.44 ; p<.01)$ : that is, in MCI patients, the more severe memory deficits were, the more life felt like a routine.

\section{DISCUSSION}

The main finding of this study is that MCI patients have alterations in the perception of the subjective passage of time, that is, they experience time as if it is passing more slowly, 
Table 3. Statements about the subjective experience of time

\begin{tabular}{|c|c|c|c|}
\hline & MCI Mean $\pm S D$ & CONTROL Mean $\pm S D$ & $p$-Value ${ }^{\mathrm{a}}$ \\
\hline 1. The past two years have been a time filled with many new experiences & $-0.06 \pm 1.62$ & $0.46 \pm 1.34$ & .07 \\
\hline 2. In the past several years my life has been quite a routine & $0.54 \pm 1.57$ & $-0.16 \pm 1.57$ & .02 \\
\hline $\begin{array}{l}\text { 3. When I think back over the past two years, few notable events came to } \\
\text { my mind }\end{array}$ & $0.07 \pm 1.72$ & $-0.11 \pm 1.67$ & .58 \\
\hline 4. There have been few notable changes in my life in the past year & $0.67 \pm 1.72$ & $0.58 \pm 1.66$ & .79 \\
\hline $\begin{array}{l}\text { 5. When I try to remember the date of some event, I often come up with a } \\
\text { time that is not as long as the true time }\end{array}$ & $0.19 \pm 1.85$ & $0.74 \pm 1.32$ & .07 \\
\hline $\begin{array}{l}\text { 6. When I think that something was just a few years ago, it often turns out } \\
\text { that it happened long before that }\end{array}$ & $0.78 \pm 1.63$ & $0.46 \pm 1.67$ & .31 \\
\hline 7. I often find that things occurred much longer ago than I thought & $0.94 \pm 1.43$ & $0.49 \pm 1.54$ & .11 \\
\hline 8. There is often not enough time to do everything I want or need to do & $0.98 \pm 1.74$ & $1.18 \pm 1.50$ & .53 \\
\hline 9. I frequently have to rush to make sure everything gets done & $0.72 \pm 1.76$ & $0.88 \pm 1.56$ & .62 \\
\hline 10. I usually have plenty of time for all things I want to accomplish in a day & $0.20 \pm 1.96$ & $0.26 \pm 1.67$ & .86 \\
\hline 11. These days I am not very busy & $0.43 \pm 2.00$ & $0.09 \pm 1.88$ & .36 \\
\hline
\end{tabular}

Note. Participants rated the statements on a seven-point scale that ranged from 'strongly disagree' [-3] to 'strongly agree' [3]. Statistically significant values are shown in bold.

a'Independent samples Student's $t$ tests.

MCI, mild cognitive impairment; $S D$, standard deviation.

compared to healthy controls. Remarkably, an abnormal internal clock was not the basis for these alterations in the perception of the subjective passage of time, since perception of the interval lengths was not different in MCI patients as compared to controls. These findings replicate a previous study, that used a prospectively time estimation paradigm, where participants were told in advance to estimate short time intervals $(10 \mathrm{~s}, 25 \mathrm{~s}, 45 \mathrm{~s}, 60 \mathrm{~s})$, and did not find changes in estimation of the interval length in MCI patients (Rueda \& Schmitter-Edgecombe, 2009). Another study asked participants to retrospectively estimate a longer time interval, the duration of an interview (administration 25 to $30 \mathrm{~min}$ ) (Heinik \& Ayalon, 2010), and again did not find changes in MCI patients, although it is worth noticing that controls in this study were psycho-geriatric referrals with cognitive complaints, not healthy participants.

The present study has the advantage to propose an extensive and detailed analysis of interval length perception, in well-characterized MCI patients and healthy controls. We used both the prospective paradigm and the retrospective

Table 4. Correlations between composite speed of time scale score and neuropsychological variables

\begin{tabular}{lcrr}
\hline \hline & & QTavg \\
\cline { 2 - 4 } & & $\mathbf{r}$ & $p$-Value \\
\hline LOGICAL MEMORY A (immediate recall) & & $\mathbf{0 . 4 5}$ & $<.01$ \\
LOGICAL MEMORY A (delayed recall) & MCI & .74 \\
& CONTROL & 0.05 & $<.01$ \\
TRAIL MAKING TEST B & MCI & $\mathbf{0 . 4 4}$ & .78 \\
GERIATRIC DEPRESSION SCALE & CONTROL & 0.04 & .05 \\
& MCI & -0.27 & .46 \\
STATE TRAIT ANXIETY INVENTORY (trace) & CONTROL & 0.10 & .11 \\
SUBJECTIVE MEMORY COMPLAINTS & MCI & .30 \\
PMRQ (prospective memory) & CONTROL & 0.22 & .32 \\
& MCI & 0.14 & .37 \\
PMRQ (retrospective memory) & CONTROL & 0.16 & 0.12 \\
& MCI & $\mathbf{0 . 3 9}$ &. $\mathbf{0 1}$ \\
& CONTROL & 0.16 & .24 \\
\hline \hline
\end{tabular}

Note. Statistically significant values are shown in bold.

MCI, mild cognitive impairment; QTavg, composite speed of time-scale score; r, Pearson's correlation; PMRQ, prospective and retrospective memory questionnaire. 
paradigm and the methods of verbal estimation and production of time intervals. No differences in prospective $(7 \mathrm{~s}, 32 \mathrm{~s}$, $58 \mathrm{~s}$ ) and retrospective (time to draw a clock, time of the interview) time estimates were found in MCI patients. No differences in prospective time intervals production $(7 \mathrm{~s}, 32 \mathrm{~s}$, $58 \mathrm{~s})$ were detected either. As mentioned, each duration in the prospective paradigm $(7 \mathrm{~s}, 32 \mathrm{~s}, 58 \mathrm{~s})$ was repeated three times $\left(1^{\text {st }}, 2^{\text {nd }}, 3^{\text {rd }}\right)$ in a pseudorandom order, both in time estimation and in time production. Again, MCI patients did not show any drift along the different periods of time, or in the order of presentation, either in time estimation or in time production, as compared to control participants.

It is interesting to note that normal subjects, in the prospective paradigm on short intervals, tend to make time estimates that are above the actual times, and produce time intervals that are under the actual times (Carrasco, Bernal, \& Redolat, 2001; Coelho et al., 2004). This finding was replicated in the present study. Usually, older adults are said to have faster internal clocks than younger adults, since they overestimate time intervals (Coelho et al., 2004; Fraisse, 1963). Regarding the age effect, it is fair to say that MCI patients do not tend either to exaggerate, or to correct, the deviations normally observed with respect to objective durations. This suggests that their internal clock is consistent with normal aging.

The subjective passage of time, as far as we know, had not been previously investigated in MCI patients. Doing so in the current study allowed to discover that MCI patients, despite their "healthy" internal clock, experience time as passing slower than controls, as shown by a significantly lower speed-of-time scale score, and had generally lower individual scores for the items related to the past. In this respect, MCI differs from normal aging, since old adults report the time to pass more quickly, rather than more slowly, when compared to young adults (Friedman \& Janssen, 2010; Wittmann \& Lehnhoff, 2005).

Results on statements about the subjective experience of time also revealed that MCI patients believed the time passing more like a routine than controls (item 2). This may reflect the forced abandonment of some complex activities of daily living that MCI experience (Pedrosa et al., 2010). From the present data, the overall level of personal activity/ engagement did not affect differently MCI patients and controls, as far as subjective time perception was concerned. The level of personal activity is known to influence time perception, as people less active tend to feel as if the time is passing more slowly (Fraisse, 1963). Thus, if MCI participants were less active due to their condition, this may had influenced their perception of time; however, if controls were also engaged in less activity, for example, due to normal aging, they might report time passage the same way as MCI patients did. Of interest, other aspects of the subject's experience of time, like forward telescoping (item 5-7) and life pressures (item 8-11), did not reveal differences between MCI patients and controls.

Since the internal clock was not found to be impaired in MCI patients, it would be important to look for other reasons that could explain the changes that MCI patients showed in the perceived speed of time. Previous studies emphasized the importance of memory and executive functions for time perception (Heinik \& Ayalon, 2010; Papagno, Allegra, \& Cardaci, 2004), as well as emotional factors, such as depression and anxiety (Nichelli, 1993). We found that the speed-of-time scale score was correlated with memory tests in MCI patients, that is, patients with more severe memory deficits felt that time was passing more slowly.

The influence of memory deficits on the distortion of perceived speed of time seems specific, since (1) it was only observed in MCI patients, not in healthy controls, and (2) speed-of-time scores correlated with memory results but not with performance in executive tests or with complaints of depression or anxiety, even if MCI patients presented abnormalities on all these measures. It is interesting to add that patients with poorer memory were also those who felt the time passing more like a routine, confirming that memory deficits are associated with alterations in the subjective experience of time. Remarkably, patients with less severe memory complaints were also those who felt time as passing most slowly. The fact that memory complaints did not correlate with memory deficits suggests that subjective memory complaints may not accurately reflect memory deficits in patients with cognitive impairment (see, for instance, Silva et al., 2014).

The relationship between memory deficits and the distortion of perceived speed of time certainly reinforces the concept, advanced by previous authors (Graf \& Grodin, 2006; Kinsbourne \& Hicks, 1990; Mimura et al., 2000; SchmitterEdgecombe \& Rueda, 2008), that memory is necessary to time perception. The idea of a connection between time perception and memory is not a modern one. It can be traced back to Aristotle, according to whom memory is what allows us to place events in time and to count them (Ricoeur, 2004). In fact, ancient philosophers like Aristotle and Saint Augustin argued that our sense of time passage is due to memory operations, although they emphasized the thought that we become acquainted of that passage by measuring time intervals, an idea that still prevails in current time studies.

Later, in the nineteenth century, philosophers and psychologists (Guyau, 1890; James, 1890) studied human temporal distortions and related them with memory changes (Guyau, 1890). However, these studies focused on the ability to count time intervals and on the quantitative aspect of time perception. Bergson was the first to drive attention toward the qualitative aspect of time perception and its association with memory (Guerlac, 2006). Indeed, Bergson famously expressed the radical thought that time is memory, a succession of qualitative states that interpenetrate and mix together (Bergson, 1889). The relevance of memory for time perception is certainly recognized by contemporary researchers, who have also called the attention to non-cognitive processes, namely qualitative sensations expressed by emotional and visceral states, that are not necessarily part of the core timekeeping system, but contribute to our self-experience and the subjective judgments of time passage (Wittmann, 2009). 
In conclusion, MCI patients experience time as passing more slowly than controls. Since time perception is an essential component of everyday goal oriented behaviors, this altered experience of time may contribute to some of the symptoms that patients with MCI report, especially memory deficits and difficulties in activities of daily life.

\section{ACKNOWLEDGMENTS}

No conflicts of interest exist. We thank all the participants, Senior University of Lisbon and Memoclínica for all the facilities provided, and Prof. Carlos João Correia for helpful suggestions. Support from Fundação para a Ciência e a Tecnologia (JPND-HC/0003/2012) is gratefully acknowledged.

\section{REFERENCES}

American Psychiatric Association. (1994). Diagnostic and Statistical Manual of Mental Disorders (4th ed.). Washington, DC: American Psychiatric Association Press.

Allman, J.M., \& Meck, W.H. (2012). Pathological distortions in time perception and timed performance, Brain, 135, 656-677.

Alzheimer's Association. (2009). Know the 10 signs. Retrieved from http://www.alz.org/national/documents/checklist_10signs.pdf

Barreto, J., Leuschner, A., Santos, F., \& Sobral, M. (2008). Escala de Depressão Geriátrica. In A. de Mendonça, \& M. Guerreiro (Eds.), Escalas e Testes na Demência (pp. 71-72). Lisboa: Grupo de Estudos de Envelhecimento Cerebral e Demência.

Bergson, H. (1889). Essai sur les données immédiates de la conscience. Ensaio sobre os dados imediatos da consciência. Portuguese edition, Lisboa: Edições 70, 2011.

Block, R.A. (1990). Models of psychological time. In R.A. Block (Ed.), Cognitive models of psychological time (pp. 1-35). Hillsdale, NJ: Lawrence Erlbaum Associates.

Buhusi, C.V., \& Meck, W.H. (2005). What makes us tick? Functional and neural mechanisms of interval timing. Nature Reviews. Neuroscience, 6, 755-765.

Castro, S.L., Cunha, L.S. \& Martins, L. (2000). Teste Stroop Neuropsicológico em Português. Série Avaliação Psicológica LFA3. Universidade do Porto: Faculdade de Psicologia e Ciências de Educação.

Carrasco, M.C., Bernal, M.C., \& Redolat, R. (2001). Time estimation and Aging: A comparison between young and elderly adults. International Journal of Aging and Human Development, 52, 91-101.

Coelho, M., Ferreira, J.J., Dias, B., Sampaio, C., Pavão Martins, I., \& Castro-Caldas, A. (2004). Assessment of time perception: The effect of aging. Journal of the International Neuropsychological Society, 10, 332-341.

Droit-Volet, S., \& Wearden, J. (2003). Les modèles d'horloge interne en psychologie du temps. L'Année Psychologique, 4, 617-654.

Fernandez, A.L., \& Marcopulos, B.A. (2008). A comparison of normative data for the Trail Making Test from several countries: Equivalence of norms and considerations for interpretation. Scandinavian Journal of Psychology, 49, 239-246.

Fortin, C., Rosseau, R., Bourque, P., \& Kirouac, E. (1993). Time estimation and concurrent non-temporal processing: Specific interference from short-term memory demands. Perception and Psychophysics, 53, 536-548.
Fraisse, P. (1963). The psychology of time. New York: Harper.

Friedman, W.J., \& Janssen, S.M.J. (2010). Aging and the speed of time. Acta Psychologica, 134, 130-141.

Folstein, M.F., Folstein, S.E., \& McHugh, P.R. (1975). Mini-Mental State. A practical method for grading the cognitive state of patients for the clinician. Journal of Psychiatric Research, 12, 189-198.

Garcia, C. (1984). Doença de Alzheimer, problemas do diagnóstico clínico. Doctoral Dissertation. Faculty of Medicine of Lisbon. Lisbon.

Ginó, S., Mendes, T., Ribeiro, F., de Mendonça, A., Guerreiro, M., \& Garcia, C. Subjective memory complaints (SMC) (2008). In A. de Mendonça, \& M. Guerreiro (Eds.), Escalas e Testes na Demência (pp. 119-120). Lisboa: Grupo de Estudos de Envelhecimento Cerebral e Demência.

Graf, P., \& Grodin, S. (2006). Time perception and time based prospective memory. In J. Glicksohn, \& M. Myslobodsky (Eds.), Timing the future. The case for time based Prospective memory (pp. 1-24). London: World Scientific Pub Co Inc.

Grodin, S. (2010). Timing and time perception: A review of recent behavioral and neuroscience findings and theoretical directions. Attention, Perception \& Psychophysics, 72, 561-582.

Guerreiro, M., Silva, A.P., Botelho, M.A., Leitão, O., CastroCaldas, A., \& Garcia, C. (1994). Adaptação à população portuguesa na tradução do 'Mini-Mental State Examination' (MMSE). Revista Portuguesa de Neurologia, 1, 9.

Guerreiro, M. (1998). Contributo da Neuropsicologia para o Estudo das Demências. Doctoral Dissertation. Faculty of Medicine of Lisbon. Lisbon.

Guerlac, S. (2006). Thinking in time. An introduction to Henri Bergson. London: Cornell University Press.

Guyau, J.M. (1890). La gènese de l'idée de temps. La mémoire et l' idée de temps, edition critique. Paris: L'Harmattan, 2011.

Heinik, J., \& Ayalon, L. (2010). Self-estimation of performance time versus actual performance time in older adults with suspected mild cognitive impairment: A clinical perspective. Israel Journal Psychiatry Related Sciences, 4, 291-296.

James, W. (1890). The principles of psychology. Cambridge, MA: Harvard University Press; 1983.

Kinsbourne, M., \& Hicks, R.E. (1990). The extended present: Evidence from time estimation by amnesics and normals. In G. Vallah, \& T. Shallice (Eds.), Neuropsychological impairments of short-term memory (pp. 319-329). Cambridge: Cambridge University Press.

Lawton, M.P., \& Brody, E.M. (1969). Assessment of older people: Self-maintaining and instrumental activities of daily living. Gerontologist, 9, 179-186.

Mangels, J.A., \& Ivry, R.B. (2001). Time perception. In B. Rapp (Ed.), Handbook of cognitive neuropsychology: What deficits recall about human mind (pp. 467-493). Hove: Psychological Press

MacLeod, C.M. (1991). Half a century of research on the Stroop effect: An integrative review. Psychological Bulletin, 109, 163-203.

Mimura, M., Kinsbourne, M., \& O’Conner, M. (2000). Time estimation by patients with frontal lobe lesions and by Korsakoff amnesics. Journal of the International Neuropsychological Society, 6, 517-528.

Nichelli, P. (1993). The neuropsychology of human temporal information processing. In F. Boller \& J. Grafman (Eds). Handbook of neuropsychology (pp. 339-371). Amsterdam: Elsevier Science Publishers B.V. 
Pantoni, L., Basile, A.M., Pracucci, J., Asplund, K., Bogousslavsky, J., Chabriat, H., ... Inzitari, D. (2005). Impact of age related cerebral white matter changes on the transition to disability - The Ladis Study: Rationale, design and methodology. Neuroepidemiology, 24, 51-62.

Papagno, C., Allegra, A., \& Cardaci, M. (2004). Time estimation abilities in Alzheimer's disease and the role of central executive. Brain and Cognition, 54, 18-23.

Pedrosa, H., Guerreiro, M., Maroco, J., Simões, M.R., Galasko, D., $\&$ de Mendonça, A. (2010). Functional evaluation distinguishes MCI patients from healthy elderly people - The ADCS/MCI/ ADL scale. Journal of Nutrition Health \& Aging, 8, 703-709.

Petersen, R.C., Smith, G.E., Waring, S.C., Ivnik, R.J., Tangalos, E.G., \& Kokmen, E. (1999). Mild cognitive impairment: Clinical characterization and outcome. Archives of Neurology, 3, 303-308.

Pouthas, V., \& Perbal, S. (2004). Time perception depends on accurate clock mechanisms as well as unimpaired attention and memory processes. Acta Neurobiologiae Experimentalis, 64, 367-385.

Reitan, R.M. (1958). Validity of the Trail Making Test as an indicator of organic brain damage. Perceptual \& Motor Skills, 8, 271-276.

Ricoeur, P. (2004). Memory, history and forgetting. Chicago: Chicago University Press.

Rueda, A.D., \& Schmitter-Edgecombe, M. (2009). Time estimation abilities in mild cognitive impairment and Alzheimer disease. Neuropsychology, 2, 178-188.

Schmand, B., Jonker, C., Hooijer, C., \& Lindeboom, J. (1996). Subjective memory complaints may announce dementia. Neurology, 46, 121-125.

Schmitter-Edgecombe, M., \& Rueda, A.D. (2008). Time estimation and episodic memory following traumatic brain injury. Journal of Clinical and Experimental Neuropsychology, 30, 212-223.
Silva, D. (2006). O inventário de Estado-Traço de Ansiedade (STAI). Avaliação psicológica: Instrumento validados para a população portuguesa, 1, 45-63.

Silva, D., Guerreiro, M., Faria, C., Maroco, J., Schmand, B.A., \& Mendonça, A.D. (2014). Significance of subjective memory complaints in the clinical setting. Journal of Geriatric Psychiatry and Neurology, 27, 259-265.

Smith, G., Della Sala, S., Logie, R.H. \& Maylor, E.A. (2000). Prospective and retrospective memory in normal aging and dementia: A questionnaire study. Memory, 8, 311-321.

Spielberger, C.D., Gorssuch, R.L., Lushene, P.R., Vagg, P.R., \& Jacobs, G.A. (1983). Manual for the state-trait anxiety inventory. Palo Alto: Consulting Psychologists Press.

Sucala, M., Scheckner, B., \& David, D. (2010). Psychological time: Interval length judgments and subjective passage of time judgments. Current Psychological Letters, 2, 1-9.

Wearden, J.H. (2005). The wrong tree: Time perception and time experience in the elderly. In J. Ducan, L. Phillips, \& P. McLeod (Eds.), Measuring the mind: Speed, age and control (pp. 134-156). Oxford: Oxford University Press.

Wittmann, M., \& Lehnhoff, S. (2005). Age effects in perception of time. Psychological Reports, 97, 921-935.

Wittmann, M. (2009). The inner experience of time. Philosophical Transactions of the Royal Society of London. Series B, Biological Sciences, 364, 1955-1967.

Yesavage, J.A., Brink, T.L., Rose, T.L., Lum, O., Huang, V., Adey, M., ... Leirer, V.O. (1982-1983). Development and validation of a geriatric depression screening scale: A preliminary report. Journal of Psychiatric Research, 1, $37-49$. 